\title{
THE DARBOUX PROPERTY AND SOLUTIONS OF ALGEBRAIC DIFFERENTIAL EQUATIONS
}

\author{
ANDrew M. BRUCKNer, Melvin Rosenfeld, ANd Lee A. Rubel
}

\author{
Dedicated to the memory of Ernst Straus
}

\begin{abstract}
The most basic notion of a solution of a differential equation is that of a function that is differentiable enough to plug into it (without demanding continuity of the derivatives of highest order) and that, of course, makes the equation a true statement when you do plug it in. The lack of continuity of the derivatives has posed many obstacles in treating these solutions. In this paper we overcome several of these obstacles in the case of algebraic differential equations by using the Darboux property of derivatives.
\end{abstract}

1. Introduction. An algebraic differential equation (ADE) is one of the form:

$$
P\left(x, y(x), y^{\prime}(x), \ldots, y^{(m)}(x)\right)=0,
$$

where $P$ is a polynomial in $m+2$ variables. We often write (1) as $P(x, \vec{y})=0$. In our context, $x$ is restricted to an open or closed interval $I$ of the real axis, and $y: I \rightarrow \mathbf{R}$ is a real-valued function. There is no real difficulty in extending some of our results (i.e. Theorems 1, 3, 4, and 5) to $y: I \rightarrow \mathbf{C}$ being a complex-valued function. An ADE in several dependent variables $y_{1}(x), \ldots, y_{n}(x)$ is similarly defined to $(1)$, but we will mention such equations only peripherally.

In discussing solutions $u(x)$ to (1) or to a system $\Sigma$ of such equations, care must be taken to enunciate how smooth $u$ is required to be-certain qualitative assertions are true for one degree of smoothness and false for another. This is the main theme of [RUB]. It is clear what we mean by analytic or $C^{\infty}$ solutions of a system $\Sigma$ of ADE's. Let us say, for convenience, that a function $u(x)$ is a basic solution of $\Sigma$ if it (a) is differentiable enough to plug into every equation of $\Sigma$ and (b) makes all these equations true statements. Following these ideas, we prove a chain of theorems with a common theme. If $u(x) \in D^{m}(I)$ (i.e. has derivatives of order up to and including order $m$ on the open interval $I$ ) then about all that can be said about $u^{(m)}(x)$ is that it is a derivative. As such, it might be discontinuous a.e. [BRU: p. 47]. It is true that it belongs to $D B_{1}$, 
i.e. has the Darboux (intermediate-value) property and is in the first class of Baire. The Darboux property can be viewed at best as a very weak form of continuity - suffice it to say that every function (even a non-measurable one) is a sum of two Darboux functions. Functions in the first class of Baire do possess a certain weak continuity property: each nonempty perfect set contains a point of relative continuity. If, however, $u$ is a $D^{m}$ solution of an ADE of order possibly less than $m$, then $u$ (and therefore $\left.u^{(m)}\right)$, must be analytic on a dense open set (Theorem 3 ). This was proved for $C^{m}$ solutions in [RUB], where a converse result [Theorem IV.2] was proved to show that the set of analyticity, for a certain fixed ADE of order 3, could be any dense open set whatsoever.

Our main result is probably the " $P-Q$ " Theorem 4. It asserts that if $u$ is $n$ times differentiable and is a basic solution of some $\mathrm{ADE}$, then $u$ will also be a basic solution of any ADE of order $n$ that it satisfies on a dense set. This can be contrasted with the fact [BRU] that there exist nonconstant differentiable functions whose derivatives vanish on a dense open set.

Our culminating result (Theorem 5) centers around a corollary of the Ritt-Raudenbush basis theorem. This corollary says that if $\Sigma$ is any system, of any cardinality, of ADE's in finitely many dependent variables $y_{1}, \ldots, y_{n}$, then there exists a finite subsystem $\Sigma_{f}$ of $\Sigma$ that has exactly the same analytic (or $C^{\infty}$, respectively) solutions that $\Sigma$ has. However it was shown in [RUB] that there exists a system $\Sigma$ of ADE's in one dependent variable $y$ such that no finite subsystem $\Sigma_{f}$ has the same basic solutions that $\Sigma$ has. Our Theorem 5 states, however, in this context of one dependent variable, that there must exist a countable subsystem $\Sigma_{\omega}$ of $\Sigma$ that has exactly the same basic solutions that $\Sigma$ has. We thank Ms. Zimmerman-Huisgen for suggesting this possibility.

\section{Statements of theorems.}

THEOREM 1. Let $a_{0}, a_{1}, \ldots, a_{n}$ be continuous functions on $[a, b] \times R^{m}$. Suppose that $y \in D^{m}[a, b]$ and satisfies on $[a, b]$ the equation:

$$
\begin{aligned}
a_{0}\left(x, y(x), \ldots, y^{(m-1)}(x)\right)+a_{1}\left(x, y(x), \ldots, y^{(m-1)}(x)\right) y^{(m)}(x) \\
+\cdots+a_{n}\left(x, y(x), \ldots, y^{(m-1)}(x)\right)\left[y^{(m)}(x)\right]^{n}=0 .
\end{aligned}
$$

If $y^{(m)}(x)$ is discontinuous at $x_{0} \in[a, b]$ then:

$$
a_{k}\left(x_{0}, y\left(x_{0}\right), \ldots, y^{(m-1)}\left(x_{0}\right)\right)=0
$$

for all $k=0,1, \ldots, n$. 
THEOREM 2. Let $Q\left(x, y_{0}, \ldots, y_{m}\right)$ be a continuous function of $m+2$ variables and suppose that $u \in D^{m}(I)$. Then

$$
q(x)=Q\left(x, u(x), u^{\prime}(x), \ldots, u^{(m)}(x)\right)
$$

has the Darboux property on I.

(Theorem 2 is not used in the sequel, but it is a pleasing result, and fits naturally in this place. Besides, the two lemmas (Lemmas 3.2.1 and 3.2.2) we provide to help us prove Theorem 2 are used several places in the proofs of the later theorems.)

THEOREM 3. Let $u$ be a basic solution of an algebraic differential equation on an interval I. Then $u$ must be analytic on a dense open subset of I.

Note. It is important to make the convention, in the case of ADE's of order 0 (i.e. algebraic equations that are not differential equations) that a basic solution is by definition continuous. Otherwise, equations like $y^{2}-\left(x^{2}+1\right)=0$ can have really terrible solutions because one could make a non-measurable choice of $\pm \sqrt{x^{2}+1}$ :

Theorem 4. (The $P$ - $Q$ theorem) Let $P$ and $Q$ be differential polynomials and let $u(x)$ be a function on the interval I such that:

(i) $u$ satisfies $P\left(x, y, y^{\prime}, \ldots, y^{(m)}\right)=0$ everywhere on $I$.

(ii) $u$ is n-times differentiable on $I$.

(iii) $u$ satisfies $Q\left(x, y, y^{\prime}, \ldots, y^{(n)}\right)=0$ on a dense subset of $I$. Then:

(iv) u satisfies $Q\left(x, y, y^{\prime}, \ldots, y^{(n)}\right)=0$ everywhere on $I$.

THEOREM 5. Let $\Sigma$ be any system, of any cardinality, of algebraic differential equations in one dependent variable $y$. Then there exists a countable subsystem $\Sigma_{\omega}$ of $\Sigma$ such that $\Sigma_{\omega}$ and $\Sigma$ have exactly the same basic solutions.

\section{Proofs of the theorems.}

3.1. Proof of Theorem 1. (By contradiction.) Suppose $y^{(m)}$ is discontinuous at $x_{0}$ but (3) is not true at $x_{0}$. Let $L=\liminf _{x \rightarrow x_{0}} y^{(m)}(x)$ and $U=\lim \sup _{x \rightarrow x_{0}} y^{(m)}(x)$. Then $L<U$. For each $x$ in $[a, b]$, define a 
polynomial $q_{x}$ by

$$
\begin{aligned}
q_{x}(w)= & a_{0}\left(x, y(x), \ldots, y^{(m-1)}(x)\right) \\
& +a_{1}\left(x, y(x), \ldots, y^{(m-1)}(x)\right) w \\
& +\cdots+a_{n}\left(x, y(x), \ldots, y^{(m-1)}(x)\right) w^{n} .
\end{aligned}
$$

By (2) we have:

$$
q_{x}\left(y^{(m)}(x)\right)=0 \text { for all } x \text { in }[a, b] .
$$

Since each $a_{k}(x)$ is continuous, and since $y(x), y^{\prime}(x), \ldots, y^{(m-1)}(x)$ are continuous, we have:

(5) $\lim _{x \rightarrow x_{0}} q_{x}=q_{x_{0}}$ uniformly on compact subsets of the complex plane $\mathbf{C}$.

Because (3) is not satisfied at $x_{0}$, the polynomial $q_{x_{0}}$ is not the zero polynomial. Let $w_{1}, \ldots, w_{p}$ be its roots, with $w_{1}=y^{(m)}\left(x_{0}\right)$. Let $N$ be a neighborhood in the plane of the set $\left\{w_{1}, \ldots, w_{p}\right\}$ such that $\bar{N}$ (the closure of $N$ ) does not contain the interval $(L, U)$, and let $T$ be a point in $(L, U) \backslash \bar{N}$. Since $y^{(m)}$ has the intermediate value property, there exists a sequence $x_{j} \rightarrow x_{0}$ so that $y^{(m)}\left(x_{j}\right) \rightarrow T$ (actually $y^{(m)}\left(x_{j}\right)=T$ ). But by (5), $\lim _{j \rightarrow \infty} q_{x_{j}}=q_{x_{0}}$ uniformly on compact subsets of $\mathbf{C}$. Hence by Hurwitz's Theorem, the roots of $q_{x}$ for large enough $j$ must be contained in $N$. Since $y^{(m)}\left(x_{j}\right) \rightarrow T \notin \bar{N}, y^{(m)}\left(x_{j}\right)$ cannot be a root of $q_{x_{j}}$ for $j$ large enough, i.e., $q_{x_{j}}\left(y^{(m)}\left(x_{j}\right)\right) \neq 0$.

This contradiction proves the theorem.

3.2. Proof of Theorem 2. (First proof.) We first state and prove two lemmas.

LEMMA 3.2.1. Let $Q\left(x, y_{0}, y_{1}, \ldots, y_{m}\right)$ be continuous on $[a, b] \times \mathbf{R}^{m+1}$, let $u \in D^{m}$, and let $q(x)=Q\left(x, u(x), u^{\prime}(x), \ldots, u^{(m)}(x)\right)$. Suppose that $q(x) \geq 0$ for all $x$ in $(a, b)$. Then $q(x) \geq 0$ for $x=a$ and $x=b$.

Proof. This follows readily from the fact that $Q$ and $x, u, \ldots, u^{(m-1)}$ are continuous, while $u^{(m)}$ has the Darboux property. For we may choose a sequence $x_{k} \downarrow a$ so that $u^{(m)}\left(x_{k}\right) \rightarrow u^{(m)}(a)$. Then $Q\left(x_{k}, u\left(x_{k}\right), \ldots, u^{(m)}\left(x_{k}\right)\right) \geq 0$. Therefore $Q\left(a, u(a), \ldots, u^{(m)}(a)\right) \geq 0$.

In what follows, if $K$ is a nonempty perfect set and if $P(x, \vec{y})=0$ is an ADE of the form (1), we may do all our analysis on $K$, and talk about 
$u$ being a basic solution on $K$ of (1). This means that all the derivatives are with respect to $K$, that is, the difference quotients are restricted to points of $K$.

LEMMA 3.2.2. Let $K$ be a nonempty perfect subset of $\mathbf{R}$ and let $u(x)$ be $n$-times differentiable with respect to $K$. Further let $u(x)$ satisfy an algebraic differential equation (1) $P=0$ of order $m$ and degree $k$ on $K$ where $m<n$. Then there exists an open interval $J$ so that $K \cap J$ is nonempty and such that $u(x)$ is infinitely differentiable with respect to $K \cap J$.

Proof. Let $P$ be the lowest polynomial with the required property. That is $P$ has the lowest order $m$, and among those of order $m, P$ has the lowest degree $k$. We let $r(x)=P\left(x, u(x), \ldots, u^{(m)}(x)\right)$, where the derivatives are taken with respect to $K$. Now $r(x) \equiv 0$ on $K$. Since $K$ is perfect, $r^{\prime}(x) \equiv 0$ on $K$. Thus:

$$
\begin{aligned}
0= & \left(D_{-1} P\right)\left(x, u, \ldots, u^{(m)}\right)+\left(D_{0} P\right)\left(x, u, \ldots, u^{(m)}\right) u^{\prime} \\
& +\left(D_{1} P\right)\left(x, u, \ldots, u^{(m)}\right) u^{\prime \prime} \\
& +\cdots+\left(D_{m} P\right)\left(x, u, \ldots, u^{(m)}\right) u^{(m+1)}
\end{aligned}
$$

where

$$
D_{-1} P=\frac{\partial P}{\partial x}, \quad D_{0} P=\frac{\partial P}{\partial y}, \quad D_{1} P=\frac{\partial P}{\partial y^{\prime}}, \ldots, D_{m} P=\frac{\partial P}{\partial y^{(m)}} .
$$

This last partial derivative is called the separant of $P$, and is denoted $S=\partial P / \partial y^{(m)}$. Now it is easy to see that $S$ is lower than $P$, and therefore there exists an $x_{0}$ in $K$ where $S\left(x_{0}\right) \neq 0$. Consequently $S(x) \neq 0$ throughout a set of the form $J \cap K$ where $J$ is an open interval that contains $x_{0}$. We can solve (*) above for $u^{(m+1)}$ as a rational function $F$ of $x, u(x), \ldots, u^{(m)}(x)$. (Notice that $u^{(m+1)}$ makes sense by our hypothesis that $m<n$ and $u \in D_{K}^{n}$.) The denominator of this rational expression is $S$. We can differentiate as many times as we please in $J \cap K$ to arrive at expressions for $u^{(m+2)}, u^{(m+3)}, \ldots$ whose denominators are powers of $S$ and hence nonvanishing.

Now to the proof of the Theorem. Suppose $q(a)<c<q(b)$ but $q \neq c$ on $(a, b)$, where $q(x)=Q\left(x, u(x), u^{\prime}(x), \ldots, u^{(m)}(x)\right)$. Let:

$$
\begin{aligned}
& A=\{x: q(x)>c\} \cap[a, b] \\
& B=\{x: q(x)<c\} \cap[a, b] \\
& D=\partial A(=\text { boundary of } A) .
\end{aligned}
$$


First of all, $D$ is a perfect set. For if $x_{0}$ were an isolated point of $D$, say $x_{0} \in A$ then the two intervals contiguous to $D$ and meeting at $x_{0}$ would both be in $A$, by Lemma 3.2.1. But then $x_{0}$ would not be a boundary point of $A$.

By Theorem $1($ (i) $\Rightarrow$ (iii)) of Chapter 0 of [BRU], there exists a point $x_{0}$ in $D$ such that the restriction of $u^{(m)}$ to $D$ is continuous at $x_{0}$, because $u^{(m)}$ is in $B_{1}$. Suppose that $x_{0}$ is in $A$ (the case $x_{0}$ in $B$ is similarly treated). Then there exists an open interval $I$ containing $x_{0}$ such that $I \cap D \subseteq A$, for $x_{0}$ is also a point of relative continuity of $q(x)$, since $Q$ is continuous.

Now let $L$ be a complementary interval to $D$ in $I$. The above argument applying Lemma 3.2.1 shows that $L \subseteq A$. This contradicts the condition that $x_{0} \in D=\partial A$, since we have now a whole neighborhood (namely $I$ ) of $x_{0}$ consisting only of points of $A$.

(Second Proof). This proof is due to C. J. Neugebauer whom we thank for his kind permission to include it here. It is a simple proof modulo the fact [NEU, Theorem 4] (also Theorem on page 104 of [BRU]) that a function $f:[0,1] \rightarrow \mathbf{R}$ belongs to $D B_{1}$ (both Baire 1 and Darboux) if and only if $f$ has the following interval function convergence property $C_{1}$.

Definition. Let $I_{0}=[0,1]$ and $f: I_{0} \rightarrow R$ and let $\{I\}$ be the collection of all nondegenerate compact subintervals of $I_{0}$. We shall use the notation $I \rightarrow x$ to denote $x$ in $I$ and $|I| \rightarrow 0$. The function $f$ is said to possess property $C_{1}$ provided that for each $I$ in $\{I\}$ there exists a value $x_{I}$ in $I^{o}$ such that $I \rightarrow x$ implies that $f\left(x_{I}\right) \rightarrow f(x)$ for each $x$ in $I$.

Now to prove our Theorem, suppose that $u \in D^{m}\left(I_{0}\right)$ say, and let $q(x)$ be defined as above. Suppose further that $x \in I_{0}$ and $I \rightarrow x$. Then $u^{(m)}\left(x_{I}\right) \rightarrow u^{(m)}(x)$ for a suitable selection $x_{I}$ in $I_{0}$, since $u^{(m)} \in\left(D B_{1}\right)$. But then, because $Q$ is continuous and because $u, u^{\prime}, \ldots, u^{(m-1)}$ are continuous, we have $q\left(x_{I}\right) \rightarrow q(x)$, and hence $q \in\left(D B_{1}\right)$ and in particular $q$ is Darboux.

3.3. Proof of Theorem 3. Since this theorem was proved in [RUB] under the added hypothesis that $u \in C^{m}$ if the order of $P$ is $m$, where $u$ satisfies (1) $P(x, \vec{y})=0$, it is enough to show that if $u \in D^{m}$ and satisfies (1), on $I$, then $u^{(m)}$ is continuous on some subinterval $J$ of $I$. As earlier, we take $P$ to be the lowest differential polynomial for which $u$ satisfies (1). Write $P(x, \vec{y})=\sum P_{k}(x, \vec{y})\left[y^{(m)}\right]^{k}$. Since $P$ is not the zero polynomial, there is some $k_{0}$ such that $P_{k_{0}}$, is not the zero polynomial. Now suppose, by way of contradiction, that $u^{(m)}(x)$ is discontinuous on a dense set $\Delta$. 
Then by Theorem 1 , at each $x_{0} \in \Delta$ we must have

$$
P_{k_{0}}\left(x_{0}, u\left(x_{0}\right), u^{\prime}\left(x_{0}\right), \ldots, u^{(m-1)}\left(x_{0}\right)\right)=0 .
$$

But $P_{k_{0}}\left(x, u(x), u^{\prime}(x), \ldots, u^{(m-1)}(x)\right)$ is a continuous function of $x$ and hence we would have

$$
P_{k_{0}}\left(x, u(x), u^{\prime}(x), \ldots, u^{(m-1)}(x)\right)=0
$$

for all $x$ in $I$, which contradicts $P$ being lowest. Of course the primary inductive step where $P$ has order 0 and degree 1 is trivial.

3.4. Proof of Theorem 4. We note that since $u$ satisfies $P=0$ everywhere, $u$ is analytic on a dense open set by Theorem 3 and because it satisfies $Q=0$ on a dense set, it must satisfy $Q=0$ on a dense open set.

Now, we may suppose without loss of generality, that $m=n$. For if $m>n$ then $u \in C^{n}$, and the conclusion is immediate. If, on the other hand, $m<n$, then we may differentiate $P$ a suitable number of times. Now let $V$ be the interior (relative to $(a, b)$ ) of the set $\{x$ : $\left.Q\left(x, u(x), \ldots, u^{(m)}(x)\right)=0\right\}$ and let $K=(a, b) \backslash V$. By Lemma 3.2.1, (or Theorem 2) $K$ is perfect relative to $(a, b)$. We wish to show that $K$ is empty. Suppose not. The set $\{x: q(x)=0\}$, where $q(x)=$ $Q\left(x, u(x), \ldots, u^{(m)}(x)\right)$, is dense in $K$ since it contains, by Lemma 3.2.1, the endpoints of the complementary intervals to $K$. Also, the set $\{x$ : $q(x) \neq 0\}$ is dense in $K$. Now $u^{(m)}$ is discontinuous (even relative to $K$ ) on $\{x: q(x) \neq 0\}$. Write

$$
P\left(x, y, y^{\prime}, \ldots, y^{(m)}\right)=\sum P_{k}\left(x, y, \ldots, y^{(m-1)}\right)\left[y^{(m)}\right]^{k}
$$

as before. By Theorem 1, all the functions

$$
\boldsymbol{\alpha}_{k}(x)=P_{k}\left(x, u(x), \ldots, u^{(m-1)}(x)\right)
$$

must vanish on the set $K$, first because they each vanish on a dense subset of $K$, and then, by continuity, on all of $K$. So, relative to $K$, there is a non-trivial differential polynomial $P_{k_{0}}$ of order $<m$ that annuls $u$. By Lemma 3.2.2, it follows that $u$ must be infinitely differentiable, relative to $K$, on some nontrivial portion $J \cap K$, where $J$ is an open interval. This contradicts the fact that $u$ is discontinuous, relative to $K$, on its dense subset $\{x: q(x) \neq 0\} \cap K$.

Notice that the same proof works if we require only that $Q$ be a continuous function of its arguments, and not necessarily a differential polynomial, but we must then suppose that $u$ satisfies $Q=0$ on a dense open set. When $Q$ is a polynomial, Theorem 3 gives us a dense open set when we have only a dense set to start with. 
3.5. Proof of Theorem 5. Given $\Sigma$, let $\Sigma_{f}$ be the finite subsystem of $\Sigma$ that has the same $C^{\infty}$ solutions as $\Sigma$ on $I$. The existence of $\Sigma_{f}$ is proved in [KAP]. Now in order to be able to plug $u(x)$ into $\Sigma, u$ must be differentiable to order $N$ for some $0 \leq N \leq \infty$. That is, the equations of $\Sigma$ demand sufficient differentiability of $u$. Enlarge $\Sigma_{f}$ to $\Sigma_{\omega}$ by throwing into $\Sigma_{\omega}$ at most a countable number of equations from $\Sigma$ so that now the differentiability demands of $\Sigma_{\omega}$ are the same as those of $\Sigma$. We claim that $\Sigma_{\omega}$ has exactly the same basic solutions as $\Sigma$. It is clear that every basic solution of $\Sigma$ is a basic solution of $\Sigma_{\omega}$. For the converse, let $u$ be a basic solution of $\Sigma_{\omega}$, and let $Q$ be a differential polynomial in $\Sigma$. Now $u$ is analytic on a dense open set $\Omega$ by Theorem 3 and since $u$ satisfies $\Sigma_{f}$ on $\Omega$, it must satisfy $Q$ on $\Omega$ since locally, every analytic solution of $\Sigma_{f}$ solves $\Sigma$. But note that $u$ is now everywhere differentiable enough to plug into $Q$, and we may apply the $P-Q$ Theorem (Theorem 4) to conclude that $u$ is a solution everywhere of $Q$.

We conclude this paper by reminding the reader that we have restricted out attention to ADE's in one dependent variable. This leaves open a number of challenging problems about systems of ADE's in a finite number of dependent variables.

\section{REFERENCES}

[BRU] Andrew M. Bruckner, Differentiation of Real Functions, Lecture Notes in Mathematics, Number 659, Springer-Verlag 1978.

[KAP] Irving Kaplansky, An introduction to Differential Algebra, second edition, Hermann, Paris, 1976.

[NEU] C. J. Neugebauer, Darboux functions of Baire class one and derivatives, Proc. Amer. Math. Soc., 13 (1962), 838-843.

[RUB] Lee A. Rubel, Solutions of algebraic differential equations, J. Differential Equations, 49 (1983), 441-452.

Received April 16, 1984. The research of the first and third authors was partially supported by grants from the National Science Foundation. The third author thanks Purdue University and the Institut des Hautes Études Scientifiques for their hospitality and support during the preparation of this paper.

UNiversity of California, SaNta Barbara

SANTA BARBara, CA 93106

AND

UNIVERSITY OF ILLINOIS

1409 WeSt GREen STREET

URBANA, IL 61801 